\title{
The severity of symptoms and outcomes of patients admitted with rheumatic diseases and COVID-19; a prospective cohort study in Iran
}

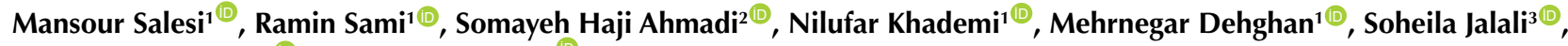 \\ Marjan Mansourian ${ }^{4}$, Huriyeh Hashemi ${ }^{*}{ }^{\circledR}$
}

${ }^{1}$ Departman of Internal Medicine, School of Medicine, Isfahan University of Medical Sciences, Isfahan, Iran

${ }^{2}$ Department of Radiology, Isfahan University of Medical Sciences, Isfahan, Iran

${ }^{3}$ Department of Epidemiology and Biostatistics, School of Health Isfahan University of Medical Sciences, Isfahan, Iran

${ }^{4}$ Pediatric Cardiovascular Research Center, Cardiovascular Research Institute, Isfahan University of Medical Sciences, Isfahan, Iran

*Correspondence to

Huriyeh Hashemi, Email:

h.hashemi@med.mui.ac.ir,

Hashemihurie@gmail.com

Received 13 Jan. 2021

Accepted 2 Mar. 2021

Published online 1 Apr. 2021

Keywords: COVID-19, Rheumatic diseases, Severity, Outcome

\begin{abstract}
Introduction: In severe COVID-19 patients, a pre-inflamatory condition leads to a cytokine storm syndrome. This may signify the need for using immunomodulatory drugs. Patients with rheumatic diseases (RDs) are prone to severe infectious diseases.

Objectives: Whether the presence of the RD itself or the use of its drugs in patients with COVID-19 increases the severity of symptoms and outcomes remains largely unknown.

Patients and Methods: In a prospective cohort study conducted in Khorshid hospital of Isfahan, Iran, 219 patients with COVID-19 were enrolled and divided into two groups of patients with a positive history for RD $(n=19)$ and those without this history (NRD, $\mathrm{n}=200$ ). The severity of symptoms and outcomes was compared between the groups.

Results: Shortness of breath $(P=0.001)$, cough $(P=0.019)$, and weakness $(P=0.001)$ were significantly higher in the RD than the NRD group after, but not before, recovery. All the patients in the RD group had comorbid diseases (hypertension, diabetes mellitus, ischemic heart disease, and cerebrovascular disease), the number of which was significantly higher than that of the NRD group $(P<0.001)$. The Charlson Comorbidity Index $(\mathrm{CCl})$ was used to predict 10-year survival in patients with multiple comorbidities. It was significantly lower in the RD group as opposed to the NRD group $(P<0.001)$. Logistic regression also showed a non-significantly higher chance of the composite outcome (ICU admission, death status, and intubation status) in the RD group as opposed to the NRD group, both before and after adjustment for confounding factors. However, no difference was found between the RD patient who received corticosteroid as a treatment (RD-CS) and those who did not (RD-NCS).

Conclusion: The results showed an increased risk for severe forms of COVID-19 in RD patients. This risk is possibly attributable to a high prevalence of comorbidities in these patients.
\end{abstract}

\section{Introduction}

In the last two decades, there have been three major global outbreaks of acute respiratory illnesses caused by coronaviruses, the most dangerous of which is the current pandemic, first observed in Wuhan, China in December 2019 (COVID-19). The disease has spread to six continents, with more than 118 million cases and over 2/6 million deaths, making it a major public health emergency in the world (1).

A pre-inflammatory condition with an increase in the IL- $1 \beta$, tumor necrosis factor- $\alpha$ (TNF- $\alpha$ ), granulocyte colony-stimulating factor (G-CSF), IP10/CXCL10, monocyte chemoattractant protein 1 (MCP-1), and macrophage inflammatory protein 1-a (MIP-

\begin{abstract}
Key point
It remains unresolved whether the rheumatic disease (RD) itself or the use of glucocorticoids and disease-modifying antirheumatic drugs in patients with concurrent rheumatic diseases and COVID-19 increases or decreases the severity of symptoms and outcome. An increased risk for severe forms of COVID-19 in patients with RD is shown in our study. This may be attributed to a high prevalence of comorbidities in these patients.
\end{abstract}

1) levels has been reported in COVID-19 patients, leading to a cytokine storm syndrome in those in need of intensive care. This may signify the need for using immunomodulatory drugs in severe cases (2). Nevertheless, patients with a suppressed

Copyright $(\odot 2021$ The Author(s); Published by Nickan Research Institute. This is an open-access article distributed under the terms of the Creative Commons Attribution License (http://creativecommons.org/licenses/by/4.0), which permits unrestricted use, distribution, and reproduction in any medium, provided the original work is properly cited. 
immune system might not be able to combat the virus properly due to the lack of immediate immunological response and are more likely to develop COVID-19 complications (3).

Evidence suggests that patients with rheumatic diseases (RDs) are prone to severe infectious diseases. However, the disease itself and RD-related genetic and environmental factors, such as susceptible genes and living habits, and the use of glucocorticoids and conventional or biologic disease-modifying antirheumatic drugs (DMARDs) may reduce host defense against infection $(4,5)$. It has been found that altered immunological function, disability, immobility, and joint surgery which occur in patients with $\mathrm{RD}$ contribute to the increased susceptibility to infections in these patients (6).

Furthermore, glucocorticoidssuchasmethylprednisolone and prednisone, and several conventional and biologic DMARDs, including hydroxychloroquine and chloroquine, have the potential to modulate the cytokine storm in patients with concurrent severe COVID-19 and rheumatoid arthritis (7). However, evidence emerging from studies is mixed and controversial, as DMARDs are shown to be associated with an increased risk of tuberculosis, forms of pneumonia and pyogenic bacterial infections, herpes zoster, and infections after orthopedic surgeries in patients with RDs (6).

\section{Objectives}

It remains to be resolved whether the presence of the disease itself or the use of glucocorticoids and DMARDs in patients with concurrent RD and COVID-19 increases or decreases the severity of symptoms and outcome. This prospective study aimed to find a proper answer to this question.

\section{Patients and Methods}

\section{Study design}

In a prospective cohort study conducted in the emergency department of Khorshid hospital of Isfahan, Iran, between February and May 2020, 219 patients with COVID-19 were enrolled using the convenience sampling method. The patients were divided into two groups of patients with a positive history for RD and those without this history (NRD), then the severity of symptoms and outcomes was compared between the groups.

The inclusion criteria were 1) definite diagnosis of COVID-19 confirmed by positive polymerase chain reaction $(\mathrm{PCR})$ test or imaging findings compatible with COVID-19 (8), and 2) informed self-consent to participate in the study. Patients were excluded from this study if they did not meet the inclusion criteria.

Upon admission, all the patients were examined by an expert rheumatologist and the presence/absence and type of RDs were determined according to the EULAR/ ACR (European League Against Rheumatism /American College of Rheumatology) criteria (https://www.eular. org). Then, the patients' demographic data such as age and gender, and clinical data including signs, symptoms, recent travel history, drug history, history of RDs, concomitant illnesses, symptom onset to admission time, hospitalization and admission to the intensive care unit (ICU) duration, and mortality rate (in- and outpatients) were recorded. Circulating biochemical factors such as complete blood count (CBC), aspartate transaminase (AST), alanine transaminase (ALT), calcium (Ca), and phosphorous $(\mathrm{P})$ levels, oxygen saturation $\left(\mathrm{SaO}_{2}\right)$, and the high-resolution computed tomography (HRCT) score, were obtained. The results were compared between the RD and NRD groups, and also between the RD patients who received corticosteroid as a treatment (RD-CS) and those who did not (RD-NCS).

The patients were followed-up by several members of the research team by phone calls 1,4 , and 12 weeks after discharge to ensure their condition (complete resolution, re-hospitalization, or death). The Charlson Comorbidity Index (CCI) was used to predict 10-year survival in patients with multiple comorbidities which was compared between the groups (9).

\section{Ethical issues}

The research followed the tenets of the Declaration of Helsinki. The Ethics Committee of Isfahan University of Medical Sciences approved this study. The Institutional Ethical Committee at Isfahan University of Medical Sciences approved all study protocols (IR.MUI.MED. REC.1399.306). Written informed consent was obtained from all the participants before any intervention. This study was based on a research project at Isfahan University of Medical Sciences (proposal\# 199140).

\section{Data analysis}

The data were analyzed using SPSS 22 (IBM Corporation, Armonk, NY). Descriptive data are shown as mean \pm standard deviation (SD) or frequencies (\%). The qualitative variables were compared using the $\chi^{2}$ test, and the Fisher's exact test was performed when the data were limited. The quantitative variables were compared using an independent t-test when the data were normally distributed; otherwise, the Mann-Whitney $U$ test was used. Logistic regression was used to identify confounding variables (Model 1) and then to determine the $95 \%$ odds ratio (OR) for the composite event (ICU admission, intubation, and death) in the RD group as opposed to the NRD group (models 2 and 3). $P<0.05$ was considered statistically significant.

\section{Results}

A total of 219 patients with COVID-19 were enrolled. Subsequently, $19(8.67 \%)$ patients were categorized into the RD group and 200 (91.32\%) patients into the NRD group. In the RD group, two (10.52\%) patients had systemic lupus erythematosus (SLE) and 17 (89.48\%) patients 
had rheumatoid arthritis (RA). The demographic data and the clinical and paraclinical findings of the RD and NRD groups are given in Table 1 . We found no significant difference in the clinical and paraclinical findings between the groups except for shortness of breath $(P=0.001)$, cough $(P=0.019)$, and weakness $(P=0.001)$ which were significantly higher in the RD group than the NRD group after, but not before, recovery. All the patients in the RD group had comorbid diseases (hypertension, diabetes mellitus, ischemic heart disease, and cerebrovascular disease), the number of which was significantly higher than that of the NRD group $(P<0.001)$. Additionally, the

Table 1. Demographic, clinical, and paraclinical findings of the patients with and without a history of rheumatologic diseases. Data are shown as mean \pm SD or numbers $(\%)$

\begin{tabular}{|c|c|c|c|c|}
\hline \multirow{2}{*}{ Variable } & \multirow{2}{*}{ Total $(n=219)$} & \multicolumn{2}{|c|}{ Group } & \multirow{2}{*}{$P$ value } \\
\hline & & RD (n = 19) & NRD $(n=200)$ & \\
\hline \multicolumn{5}{|l|}{ Demographic data } \\
\hline Age (years) & $54.41 \pm 16.41$ & $58.26 \pm 12.77$ & $54.05 \pm 16.70$ & 0.286 \\
\hline Gender (male) & $144(65.8 \%)$ & $5(26.3 \%)$ & $139(69.5 \%)$ & 0.001 \\
\hline \multicolumn{5}{|l|}{ Laboratory Findings (before recovery) } \\
\hline White blood cell count (cells/ $\mu g$ ) & $5778.10 \pm 2783.86$ & $5925.00 \pm 2398.19$ & $5765.99 \pm 2818.40$ & 0.827 \\
\hline Lymphocyte (\%) & $20.81 \pm 9.63$ & $22.86 \pm 9.64$ & $20.64 \pm 9.64$ & 0.379 \\
\hline Lymphocyte (categorized, <1000 cells/ug) & $21(9.6 \%)$ & $0(0.0 \%)$ & $21(10.5 \%)$ & 0.380 \\
\hline Platelet count (cells/ $\mu \mathrm{g})$ & $184.35 \pm 68.84$ & $213.25 \pm 85.65$ & $181.97 \pm 66.98$ & 0.081 \\
\hline $\operatorname{ALT}(\mathrm{U} / \mathrm{L})$ & $33.87 \pm 31.66$ & $27.41 \pm 13.10$ & $34.49 \pm 32.85$ & 0.380 \\
\hline $\mathrm{ALT}>60(\mathrm{U} / \mathrm{L})$ & $20(9.1 \%)$ & $0(0.0 \%)$ & $20(10.0 \%)$ & 0.226 \\
\hline $\operatorname{AST}(U / L)$ & $46.53 \pm 33.40$ & $44.00 \pm 21.37$ & $46.78 \pm 34.37$ & 0.744 \\
\hline AST > 40 (U/L) & $94(42.9 \%)$ & $9(47.4 \%)$ & $85(42.5 \%)$ & 0.801 \\
\hline $\mathrm{Ca}(\mathrm{mg} / \mathrm{dL})$ & $8.50 \pm 0.76$ & $8.72 \pm 0.99$ & $8.47 \pm 0.73$ & 0.196 \\
\hline $\mathrm{P}(\mathrm{mg} / \mathrm{dL})$ & $2.91 \pm 0.73$ & $3.01 \pm 0.63$ & $2.90 \pm 0.74$ & 0.550 \\
\hline $\mathrm{SaO}_{2}(\%)$ & $89.69 \pm 7.02$ & $89.95 \pm 5.08$ & $89.67 \pm 7.18$ & 0.868 \\
\hline $\mathrm{SaO}_{2}$ (categorized, $<90 \%$ ) & $73(33.3 \%)$ & $6(31.6 \%)$ & $67(33.5 \%)$ & 0.998 \\
\hline \multicolumn{5}{|l|}{ Laboratory Findings (after recovery) } \\
\hline White blood cell count (cells/ug) & $7395.38 \pm 4394.80$ & $6462.50 \pm 3810.86$ & $7243.44 \pm 4439.96$ & 0.628 \\
\hline Lymphocyte (\%) & $21.70 \pm 12.82$ & $25.74 \pm 13.16$ & $21.43 \pm 12.81$ & 0.359 \\
\hline Lymphocyte (categorized, <1000 cells/ug) & $31(14.2 \%)$ & $1(5.3 \%)$ & $30(15.0 \%)$ & 0.679 \\
\hline Platelet count & $204.75 \pm 87.88$ & $196.00 \pm 111.17$ & $205.32 \pm 86.67$ & 0.773 \\
\hline ALT & $72.83 \pm 45.78$ & $56.33 \pm 44.43$ & $74.33 \pm 46.27$ & 0.522 \\
\hline ALT (categorized, >60) & $19(8.7 \%)$ & $1(5.3 \%)$ & $18(9.0 \%)$ & 0.593 \\
\hline AST & $57.94 \pm 32.77$ & $69.25 \pm 58.96$ & $56.58 \pm 29.39$ & 0.473 \\
\hline AST (categorized, >40) & $27(12.3 \%)$ & $3(15.8 \%)$ & $24(12.0 \%)$ & 0.998 \\
\hline $\mathrm{Ca}$ & $8.34 \pm 0.79$ & $8.89 \pm 1.87$ & $8.28 \pm 0.57$ & 0.055 \\
\hline$P$ & $3.17 \pm 0.81$ & $3.01 \pm 0.38$ & $3.19 \pm 0.85$ & 0.409 \\
\hline $\mathrm{SaO} 2$ & $92.60 \pm 3.53$ & $93.68 \pm 3.73$ & $92.48 \pm 3.49$ & 0.159 \\
\hline $\mathrm{SaO} 2$ (categorized, <90) & $22(10.0 \%)$ & $1(5.3 \%)$ & $21(10.5 \%)$ & 0.702 \\
\hline \multicolumn{5}{|l|}{ Signs and symptoms (before recovery) } \\
\hline Fever & $164(74.9 \%)$ & $11(57.9 \%)$ & $153(76.5 \%)$ & 0.079 \\
\hline Cough & $172(78.5 \%)$ & $11(57.9 \%)$ & $161(80.5 \%)$ & 0.053 \\
\hline Shortness of Breath & $139(63.5 \%)$ & $10(52.6 \%)$ & $129(64.5 \%)$ & 0.593 \\
\hline Rhinorrhea & $24(11.0 \%)$ & $3(15.8 \%)$ & $21(10.5 \%)$ & 0.452 \\
\hline Sore throat & $44(20.1 \%)$ & $5(26.3 \%)$ & $39(19.5 \%)$ & 0.569 \\
\hline Weakness & $123(56.2 \%)$ & $11(57.9 \%)$ & $112(56.0 \%)$ & 0.998 \\
\hline \multicolumn{5}{|l|}{ Signs and symptoms (after recovery) } \\
\hline Fever & $1(0.5 \%)$ & $1(5.3 \%)$ & $0(0.0 \%)$ & 0.061 \\
\hline Cough & $4(1.8 \%)$ & $2(10.5 \%)$ & $2(1.0 \%)$ & 0.019 \\
\hline Shortness of breath & $5(2.3 \%)$ & $5(26.3 \%)$ & $0(0.0 \%)$ & 0.001 \\
\hline Rhinorrhea & $0(0.0 \%)$ & $0(0.0 \%)$ & $0(0.0 \%)$ & 0.694 \\
\hline
\end{tabular}


Table 1. Continued

\begin{tabular}{|c|c|c|c|c|}
\hline \multirow{2}{*}{ Variable } & \multirow{2}{*}{ Total $(n=219)$} & \multicolumn{2}{|c|}{ Group } & \multirow{2}{*}{$P$ value } \\
\hline & & RD (n = 19) & NRD $(n=200)$ & \\
\hline Weakness & $4(1.8 \%)$ & $3(15.8 \%)$ & $1(0.5 \%)$ & 0.001 \\
\hline Temperature before recovery & $37.57 \pm 1.03$ & $37.38 \pm 0.83$ & $37.62 \pm 1.06$ & 0.361 \\
\hline Temperature before recovery (categorized, $>38$ ) & $33(15.1 \%)$ & $4(21.1 \%)$ & $29(14.5 \%)$ & 0.587 \\
\hline Temperature after recovery & $36.78 \pm 0.57$ & $36.64 \pm 0.47$ & $36.79 \pm 0.57$ & 0.303 \\
\hline Hospitalization duration (days) & $6.42 \pm 6.02$ & $7.74 \pm 5.10$ & $6.29 \pm 6.02$ & 0.319 \\
\hline Admission to ICU (yes) & $36(16.4 \%)$ & $2(10.5 \%)$ & $34(17.0 \%)$ & 0.746 \\
\hline HRCT score & $8.66 \pm 5.01$ & $10.10 \pm 4.95$ & $8.50 \pm 5.01$ & 0.319 \\
\hline HRCT score (categorized, $>6$ ) & $76(34.7 \%)$ & $9(47.4 \%)$ & $67(33.5 \%)$ & 0.501 \\
\hline HRCT score (categorized, $>7$ ) & $71(32.4 \%)$ & $8(42.1 \%)$ & $63(31.5 \%)$ & 0.747 \\
\hline Symptoms duration before admission (days) & $7.53 \pm 5.20$ & $8.63 \pm 7.36$ & $7.41 \pm 4.95$ & 0.489 \\
\hline Mortality rate (yes) & $17(7.8 \%)$ & $1(5.3 \%)$ & $16(8.0 \%)$ & 0.998 \\
\hline Smoking (yes) & $17(7.8 \%)$ & $2(10.5 \%)$ & $15(7.5 \%)$ & 0.879 \\
\hline Influenza vaccine (yes) & $4(1.8 \%)$ & $1(5.3 \%)$ & $3(1.5 \%)$ & $<0.001$ \\
\hline Comorbidity disease * (yes) & $19(8.7 \%)$ & $19(100 \%)$ & $0(0.0 \%)$ & $<0.001$ \\
\hline $\mathrm{CCl}$ & $1.30 \pm 1.52$ & $0.69 \pm 0.31$ & $1.36 \pm 1.58$ & $<0.001$ \\
\hline
\end{tabular}

HTN, HF, IHD, CVD, COPD, Asthma, Lung Fibrosis, Diabetes, Immune-compromised, Cancer, CKD, Dialysis, HIV, Organ transplant, Chemotherapy, HLP, ESRD, Hypothyroidism. HTN, hypertension; HF, heart failure; CVD, cardiovascular disease; COPD, chronic obstructive pulmonary disease; CKD, chronic kidney disease; HIV, human immunodeficiency virus; HLP, hyperlipidemia; ESRD, end-stage renal disease; RD, rheumatologic disease; NRD, no rheumatologic disease; ALT, alkaline transaminase; AST, aspartate transaminase; Ca, calcium; P, phosphate; Sa, saturation; ICU, intensive care unit; HRCT, high-resolution computed tomography; $\mathrm{CCl}$, Charlson comorbidity index.

CCI was significantly lower in the RD group as opposed to the NRD group $(P<0.001)$. Besides, the number of patients who received influenza vaccination was remarkably low in the RD group than the NRD group $(P<0.001)$.

We found no difference in the radiologic findings of patients in the RD and NRD groups except for distribution patterns (upper versus lower, $P=0.008$ ) and peripheral versus central $(P=0.001$; Table 2$)$.

Further, the analysis showed no difference in terms of demographic, clinical, and paraclinical findings between the RD-CS and RD-NCS groups (Table 3).

We supposed three logistic regression models, the response variable being the composite event (the combination of death, ICU admission, and intubation status) in all of them. In the first model, the chance of occurrence of at least one of the events was found to be almost $90 \%$ higher in the patients of the RD group than those in the NRD group. According to the second model and by eliminating the confounding effects of age and gender, the chance was almost doubled. By adding other confounding variables to the model described above, this chance was $13 \%$ higher in the RD group than in the NRD group (Table 4). However, none of these changes was significant.

\section{Discussion}

In our study, a majority of RD patients (89.48\%) had RA. This study showed that dyspnea, cough, and weakness were significantly higher in the $\mathrm{RD}$ group than in the NRD group. The results indicated that patients with RD had a significantly higher number of comorbid diseases compared with the NRD. Our findings also revealed that the CCI was remarkably higher in the $\mathrm{RD}$ group than the NRD group, predicting lower 10 -year survival rates in the former. Further, logistic regression demonstrated a nonsignificantly higher chance of the composite outcome (ICU admission, death status, and intubation status) in the $\mathrm{RD}$ group as opposed to the NRD group, both before and after adjustment for confounding factors. However, no difference was found between the RD-CS and RD-NCS groups in this regard.

Patients with concurrent RD and COVID-19 are in need of distinct medical attention; manifestations of COVID-19 may mimic those of $\mathrm{RD}$, and these patients are at risk for serious infections because of their immunosuppressed condition caused by their underlying immune state and the use of glucocorticoids and DMARDs (10). Furthermore, several medications frequently used for the treatment of RDs such as hydroxychloroquine (11), and also those which target interleukin (IL)-6 (e.g., tocilizumab, sarilumab) and IL-1 (e.g., anakinra) are being evaluated in patients with COVID-19 due to their ability to modulate cytokine storm that signals a poorer outcome in these patients (12). The question, however, remains unresolved whether $\mathrm{RD}$ patients with immunosuppressive medications are at risk for severe acute respiratory syndrome coronavirus 2 (SARS-CoV-2) infection.

Similar to our findings, in a study by Cheng et al on five patients with concomitant $\mathrm{RD}$ (one systemic sclerosis and 4 RA) and COVID-19, fever (80\%), cough (80\%), dyspnea (40\%), and fatigue (20\%) were the most frequently detected symptoms. Four patients (80\%) had a 
Table 2. Radiologic findings of the patients in both groups

\begin{tabular}{|c|c|c|c|c|}
\hline \multirow{2}{*}{ Radiologic findings } & \multirow{2}{*}{ Total $(n=219)$} & \multicolumn{2}{|c|}{ Group } & \multirow{2}{*}{$P$ value } \\
\hline & & $\operatorname{RD}(n=19)$ & NRD $(n=200)$ & \\
\hline \multicolumn{5}{|c|}{ Ground-glass opacification } \\
\hline Negative & $70(32.0 \%)$ & $8(42.1 \%)$ & $62(31.0 \%)$ & \multirow[t]{2}{*}{0.744} \\
\hline Positive & $38(17.4 \%)$ & $3(15.8 \%)$ & $35(17.5 \%)$ & \\
\hline \multicolumn{5}{|c|}{ Ground-glass consolidation } \\
\hline Negative & $53(24.2 \%)$ & $5(26.3 \%)$ & $48(24.0 \%)$ & \multirow[t]{2}{*}{0.761} \\
\hline Positive & $52(23.7 \%)$ & $6(31.6 \%)$ & $46(23.0 \%)$ & \\
\hline \multicolumn{5}{|l|}{ Consolidation } \\
\hline Negative & $90(41.1 \%)$ & $10(52.6 \%)$ & $80(40.0 \%)$ & \multirow{2}{*}{0.998} \\
\hline Positive & $14(6.4 \%)$ & $1(5.3 \%)$ & $13(6.5 \%)$ & \\
\hline \multicolumn{5}{|l|}{ Centrilobular nodule } \\
\hline Negative & $103(47.0 \%)$ & $10(52.6 \%)$ & $93(46.5 \%)$ & \multirow[t]{2}{*}{0.320} \\
\hline Positive & $3(1.4 \%)$ & $1(5.3 \%)$ & $2(1.0 \%)$ & \\
\hline \multicolumn{5}{|c|}{ Bronchial wall thickening } \\
\hline Negative & $99(45.2 \%)$ & $10(52.6 \%)$ & $89(44.5 \%)$ & \multirow[t]{2}{*}{0.748} \\
\hline Positive & $7(3.2 \%)$ & $1(5.3 \%)$ & $6(3.0 \%)$ & \\
\hline \multicolumn{5}{|l|}{ Reticular } \\
\hline Negative & $70(32.0 \%)$ & $5(26.3 \%)$ & $65(32.5 \%)$ & \multirow[t]{2}{*}{0.280} \\
\hline Positive & $35(16.0 \%)$ & $6(31.6 \%)$ & $29(14.5 \%)$ & \\
\hline \multicolumn{5}{|l|}{ Lymphadenopathy } \\
\hline Negative & $105(48.0 \%)$ & $11(57.9 \%)$ & $94(47.0 \%)$ & \multirow[t]{2}{*}{0.998} \\
\hline Positive & $0(0.0 \%)$ & $0(0.0 \%)$ & $0(0.0 \%)$ & \\
\hline \multicolumn{5}{|l|}{ Vascular enlargement } \\
\hline Negative & $94(42.9 \%)$ & $10(52.6 \%)$ & $84(42.0 \%)$ & \multirow[t]{2}{*}{0.728} \\
\hline Positive & $10(4.6 \%)$ & $1(5.3 \%)$ & $9(4.5 \%)$ & \\
\hline \multicolumn{5}{|l|}{ Pleural effusion } \\
\hline Negative & $104(47.5 \%)$ & $11(57.9 \%)$ & $93(46.5 \%)$ & \multirow[t]{2}{*}{0.648} \\
\hline Positive & $2(0.9 \%)$ & $0(0.0 \%)$ & $2(1.0 \%)$ & \\
\hline \multicolumn{5}{|l|}{ Distribution } \\
\hline None & $36(16.4 \%)$ & $5(26.3 \%)$ & $31(15.5 \%)$ & \multirow{3}{*}{0.008} \\
\hline Upper & $6(2.7 \%)$ & $1(5.3 \%)$ & $5(2.5 \%)$ & \\
\hline Lower & $66(30.1 \%)$ & $5(26.3 \%)$ & $61(30.5 \%)$ & \\
\hline \multicolumn{5}{|l|}{ Transverse } \\
\hline None & $24(10.9 \%)$ & $4(21.1 \%)$ & $20(10.0 \%)$ & م001 \\
\hline Peripheral & $64(29.2 \%)$ & $5(26.3 \%)$ & $59(29.5 \%)$ & 0.001 \\
\hline Central & $4(1.8 \%)$ & $2(10.5 \%)$ & $2(1.0 \%)$ & \\
\hline Region & & & & \\
\hline Unilateral & $16(7.3 \%)$ & $1(5.3 \%)$ & $15(7.5 \%)$ & 0.787 \\
\hline Bilateral & $89(40.6 \%)$ & $10(52.6 \%)$ & $79(39.5 \%)$ & \\
\hline Scattered & & & & \\
\hline Focal & $9(4.1 \%)$ & $1(5.3 \%)$ & $8(4.0 \%)$ & \\
\hline Multifocal & $95(43.4 \%)$ & $10(52.6 \%)$ & $85(42.5 \%)$ & נד. \\
\hline Diffuse & $2(0.9 \%)$ & $0(0.0 \%)$ & $2(1.0 \%)$ & \\
\hline
\end{tabular}

RD, rheumatologic disease; NRD, no rheumatologic disease. Data are shown as number (\%).

positive history of other comorbid diseases (most notably hypertension). This study showed that RD patients were more likely to have severe (one-fifth) or critical (twofifths) COVID-19 illness. These patients had higher levels of IL-1 and IL-6 compared with others, indicating a need for the management of cytokine storm and immune regulation using biological agents such as IL- 6 blocker (IVIg, $400 \mathrm{mg}$ ) and plasma exchange $(400 \mathrm{~mL}$, once or twice) in patients with severe and critical COVID-19 (13). In this light, although the administration of synthetic and biologic DMARDs is linked to a possible further rise in the incidence of life-threatening infections, the poor management of RA activity is a more important infectious risk factor in patients with $\mathrm{RD}$. Thus, it has been suggested that RA patients should be encouraged to adhere to their medical regimen even during the COVID-19 pandemic. This may decrease a subsequent COVID-19-induced rise in the disease flares, patient burden, disability, poor quality of life, and seeking healthcare attention (14). However, the study by Chen et al was performed on a limited number of patients without a control arm to compare these findings with COVID-19 patients without RD.

Similarly, in a study conducted by Mathian et al on 17 patients with SLE, an increased risk for severe forms 
Table 3. Demographic, clinical, and paraclinical findings of the RD patients with and without history corticosteroid treatment

\begin{tabular}{|c|c|c|c|}
\hline \multirow{2}{*}{ Variable } & \multicolumn{2}{|c|}{ Groups } & \multirow{2}{*}{$P$ value } \\
\hline & RD-CS $(n=10)$ & RD-NCS $(n=9)$ & \\
\hline Age (years) & $57.50 \pm 9.66$ & $59.11 \pm 16.14$ & 0.792 \\
\hline Gender (male) & $2(20.0 \%)$ & $3(33.3 \%)$ & 0.628 \\
\hline \multicolumn{4}{|l|}{ Laboratory findings } \\
\hline 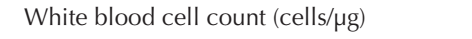 & $5588.89 \pm 2859.83$ & $6357.14 \pm 1756.76$ & 0.544 \\
\hline Lymphocyte (\%) & $21.42 \pm 11.67$ & $24.70 \pm 6.61$ & 0.519 \\
\hline Platelet count (cells/ $\mu g$ ) & $213.78 \pm 76.38$ & $212.57 \pm 102.76$ & 0.979 \\
\hline $\operatorname{ALT}(\mathrm{U} / \mathrm{L})$ & $23.88 \pm 9.63$ & $31.37 \pm 15.88$ & 0.251 \\
\hline AST (U/L) & $42.11 \pm 19.78$ & $46.13 \pm 24.22$ & 0.712 \\
\hline $\mathrm{Ca}(\mathrm{mg} / \mathrm{dL})$ & $8.92 \pm 0.71$ & $8.46 \pm 1.28$ & 0.347 \\
\hline $\mathrm{P}(\mathrm{mg} / \mathrm{dL})$ & $3.16 \pm 0.56$ & $2.81 \pm 0.69$ & 0.248 \\
\hline $\mathrm{SaO}_{2}(\%)$ & $91.50 \pm 1.78$ & $88.22 \pm 6.94$ & 0.202 \\
\hline \multicolumn{4}{|l|}{ Signs and symptoms } \\
\hline Fever & $5(50.0 \%)$ & $6(66.7 \%)$ & 0.367 \\
\hline Cough & $6(60.0 \%)$ & $5(55.6 \%)$ & 0.998 \\
\hline Shortness of breath & $4(40.0 \%)$ & $6(66.7 \%)$ & 0.637 \\
\hline Rhinorrhea & $2(20.0 \%)$ & $1(11.1 \%)$ & 0.998 \\
\hline Sore throat & $2(20.0 \%)$ & $3(33.3 \%)$ & 0.593 \\
\hline Weakness & $6(60.0 \%)$ & $5(55.6 \%)$ & 0.604 \\
\hline Temperature $\left({ }^{\circ} \mathrm{C}\right)$ & $37.34 \pm 0.87$ & $37.42 \pm 0.82$ & 0.836 \\
\hline HRCT score & $11.13 \pm 4.67$ & $7.33 \pm 5.51$ & 0.280 \\
\hline Symptoms duration before admission (days) & $7.90 \pm 4.70$ & $9.44 \pm 9.77$ & 0.661 \\
\hline Smoking (yes) & $1(10.0 \%)$ & $1(11.1 \%)$ & 0.998 \\
\hline Influenza vaccine (yes) & $1(10.0 \%)$ & $0(0.0 \%)$ & 0.478 \\
\hline Comorbidity disease* (yes) & $10(100.0 \%)$ & $9(100.0 \%)$ & - \\
\hline $\mathrm{CCl}$ & $0.59 \pm 0.37$ & $0.78 \pm 0.19$ & 0.143 \\
\hline Hospitalization duration (days) & $8.50 \pm 7.25$ & $6.89 \pm 4.51$ & 0.574 \\
\hline Admission to ICU (yes) & $1(10.0 \%)$ & $1(11.1 \%)$ & 0.998 \\
\hline Intubation status (yes) & $0(0.0 \%)$ & $1(11.1 \%)$ & 0.474 \\
\hline Mortality rate (yes) & $0(0.0 \%)$ & $1(11.1 \%)$ & 0.474 \\
\hline
\end{tabular}

*HTN, HF, IHD, CVD, COPD, asthma, lung fibrosis, diabetes, immune-compromised, cancer, CKD, Dialysis, HIV, organ transplant, chemotherapy, HLP, ESRD, hypothyroidism.

HTN, hypertension; HF, heart failure; CVD, cardiovascular disease; COPD, chronic obstructive pulmonary disease; CKD, chronic kidney disease; HIV, human immunodeficiency virus; HLP, hyperlipidemia; ESRD, end-stage renal disease; RD, rheumatologic disease; NRD, no rheumatologic disease; ALT, alkaline transaminase; AST, aspartate transaminase; Ca, calcium; P, phosphate; Sa, saturation; ICU, intensive care unit; HRCT, high-resolution computed tomography; CCI, Charlson comorbidity index.

Data are shown as mean \pm SD or number $(\%)$.

Table 4. Estimated ORs and $95 \%$ Cls by logistic regression models for the composite outcome (ICU admission, death status, and intubation status)

\begin{tabular}{|c|c|c|c|c|c|}
\hline Model $1^{*}$ & & Model $2^{*}$ & & Model $3^{*}$ & \\
\hline OR $(95 \% \mathrm{Cl})$ & $P$ value & OR $(95 \% \mathrm{Cl})$ & $P$ value & OR $(95 \% \mathrm{Cl})$ & $P$ value \\
\hline $1.929(0.427,8.717)$ & 0.393 & $2.142(0.453,10.130)$ & 0.336 & $1.135(0.220,5.862)$ & 0.880 \\
\hline
\end{tabular}

OR, odds ratio. *Model 1: unadjusted, Model 2: adjusted by age and sex, and Model 3: adjusted by other significant covariates indicated in Table 1.

of COVID-19 was detected. This risk was attributed to a high prevalence of comorbidities such as chronic kidney disease and obesity in patients with SLE (15). In a largescale study by Gianfrancesco et al on 600 cases (RA (230, 38\%), SLE (85, 14\%), and psoriatic arthritis (74, $12 \%)$ ) from 40 countries, almost half of the cases were hospitalized $(277,46 \%)$ and 55 (9\%) died (higher than the general population). The most frequent comorbidities were hypertension $(199,33 \%)$, lung disease $(127,21 \%)$, diabetes $(69,12 \%)$, and cardiovascular disease $(63,11 \%)$. Similar to our study, patients with comorbidities such as hypertension, cardiovascular disease, and diabetes had higher odds of hospitalization. This study found that glucocorticoid exposure of $\geq 10 \mathrm{mg} / \mathrm{d}$ was linked to a higher and anti-TNF with lower hospitalization rates in RD patients. The use of DMARDs or non-steroidal antiinflammatory drugs was not associated with an increased risk of hospitalization (16). In our study, however, no 
difference was detected in paraclinical and clinical findings between the RD-CS and RD-NCS patients.

In a cohort study conducted on 110 patients with concomitant RD and COVID-19, the most common underlying RDs were RA (36\%), psoriatic arthritis (17\%), and SLE (17\%), respectively. The most frequently presented symptoms were fever (79\%), cough (77\%), and dyspnea (50\%). The mortality rate due to COVID-19 was $5 \%$, which was almost equal to that of the general population $(5.2 \%)$. The five most common comorbid conditions in the deceased patients were hypertension (28\%), lung disease $(20 \%)$, cardiovascular disease (11\%), morbid obesity (8\%), and diabetes (8\%). The results of this study showed no increased risk of death due to COVID-19 in patients with RD compared with those without RD (17). However, this study lacked a proper control arm and was not able to compare the clinical and paraclinical findings with the general population affected by COVID-19. In line with this, Monti et al assessed the clinical course of COVID-19 in 320 patients (68\% female, mean age $55 \pm 14$ years) treated with DMARDs (57\% with rheumatoid arthritis, $43 \%$ with spondyloarthritis, 52\% treated with TNF inhibitors, $48 \%$ with other DMARDs). This study showed that RD patients under treatment with DMARDs were not at an increased risk of respiratory or life-threatening complications from SARS-CoV-2 compared with the general population. This might have resulted from the immunomodulatory effects of these treatments in RD patients with COVID-19 who had an aberrant inflammatory and cytokine response induced by the host immune system (18). Similarly, evidence obtained from large population-based studies demonstrated no increased risk of severe COVID-19 disease in patients with RD compared with those with other comorbidities $(19,20)$. These findings are in line with the reports published in SARS and Middle East respiratory syndrome outbreaks, showing that immunocompromised patients with organ transplantation, cancer, or autoimmune diseases are not at an increased risk for coronavirus-induced death and complications $(21,22)$.

Our study had several potential shortcomings that may have affected its outcomes. First, this study was performed on a limited number of patients. Studies with a higher number of RD patients are strictly encouraged. The problem could be tackled by the COVID-19 Global Rheumatology Alliance which helps the generation of rapid data on patients with $\mathrm{RD}$ and those using immunemodulating therapies. Second, our study may have suffered from selection bias as patients with more severe diseases are most likely to be captured. Thus, patients with a mildto-moderate course of the disease are lost.

\section{Conclusion}

Our results demonstrated that patients with $\mathrm{RD}$ had a significantly higher number of comorbid diseases compared with the NRD. As a result, the CCI was significantly and the composite outcome (ICU admission, death status, and intubation status) was non-significantly higher in the $\mathrm{RD}$ group than the NRD group, both before and after adjustment for confounding factors. Nevertheless, no difference was found between the RD-CS and RD-NCS groups in this regard. These findings should be validated in larger case-control studies.

Limitations of the study

The limitation of our study was its small sample size in the RD group.

\section{Authors' contribution}

MS, RS, and $\mathrm{HH}$ contributed to the conception and design of the study. MS, RS, SHA, NKH, MD, SJ, MM, and HH participated in the collection of data and drafting the work. MS, RS, and $\mathrm{HH}$ contributed to revising the work. All the authors have approved the final version submitted for publication and take responsibility for the statements made in the published article.

Conflicts of interest

The authors declare that they have no competing interests.

Ethical considerations

Ethical considerations (lack of plagiarism, data fabrication, and double publication) have been completely observed by the authors.

\section{Funding/Support}

This research was financially supported by Isfahan University of Medical Sciences (Grant\# 199140)

\section{References}

1. Yang C, Qiu X, Fan H, Jiang M, Lao X, Zeng Y, et al. Coronavirus disease 2019: reassembly attack of coronavirus. Int J Environ Health Res. 2020:1-9.doi: 10.1080/09603123.2020.1747602

2. Perricone C, Triggianese P, Bartoloni E, Cafaro G, Bonifacio $A F$, Bursi $R$, et al. The anti-viral facet of anti-rheumatic drugs: lessons from COVID-19. J Autoimmun. 2020:102468. doi: 10.1016/j.jaut.2020.102468

3. Novi G, Mikulska M, Briano F, Toscanini F, Tazza F, Uccelli A, et al. COVID-19 in a MS patient treated with ocrelizumab: does immunosuppression have a protective role? Mult Scler Relat Disord. 2020;42:102120. doi: 10.1016/j.msard.2020.102120.

4. Li S, Yu Y, Yue Y, Zhang Z, Su K. Microbial infection and rheumatoid arthritis. J Clin Cell Immunol. 2013;4:174. doi: 10.4172/2155-9899.1000174

5. Fragoulis GE, Sipsas NV. When rheumatology and infectious disease come together. London, England: SAGE Publications Sage; 2019.

6. Atzeni F, Masala IF, Di Franco M, Sarzi-Puttini P. Infections in rheumatoid arthritis. Curr Opin Rheumatol. 2017;29:323-30. doi: 10.1097/bor.0000000000000389

7. Lu C, Li S, Liu Y. Role of immunosuppressive therapy in rheumatic diseases concurrent with COVID-19. Ann Rheum Dis. 2020;79:737-9.doi: 10.1136/annrheumdis-2020-217460

8. Long C, Xu H, Shen Q, Zhang X, Fan B, Wang C, et al. Diagnosis of the Coronavirus disease (COVID-19): rRT-PCR or CT? Eur J Radiol. 2020;126:108961. doi: 10.1016/j.ejrad.2020.108961

9. Charlson ME, Pompei P, Ales KL, MacKenzie CR. A new method of classifying prognostic comorbidity in longitudinal studies: development and validation. J Chronic Dis. 1987;40:373-83. doi: 10.1016/0021-9681(87)90171-8.

10. Kourbeti IS, Ziakas PD, Mylonakis E. Biologic therapies in rheumatoid arthritis and the risk of opportunistic infections: a 
meta-analysis. Clin Infect Dis. 2014;58:1649-57.doi: 10.1093/ cid/ciu185

11. Kim AHJ, Sparks JA, Liew JW, Putman MS, Berenbaum F, Duarte-García A, et al. A Rush to Judgment? Rapid Reporting and Dissemination of Results and Its Consequences Regarding the Use of Hydroxychloroquine for COVID-19. Ann Intern Med. 2020;172:819-821. doi: 10.7326/M20-1223.

12. Mehta P, McAuley DF, Brown M, Sanchez E, Tattersall RS, Manson JJ, et al. COVID-19: consider cytokine storm syndromes and immunosuppression. Lancet (London, England). 2020;395:1033. doi: 10.1016/S0140-6736(20)30628-0

13. Cheng C, Li C, Zhao T, Yue J, Yang F, Yan Y, et al. COVID-19 with rheumatic diseases: a report of 5 cases. Clin Rheumatol. 2020:1-5. doi: 10.1007/s10067-020-05160-x

14. Favalli EG, Ingegnoli F, De Lucia O, Cincinelli G, Cimaz R, Caporali R. COVID-19 infection and rheumatoid arthritis: Faraway, so close! Autoimmun Rev. 2020;19:102523. doi: 10.1016/j.autrev.2020.102523

15. Mathian A, Mahevas M, Rohmer J, Roumier M, Cohen-Aubart F, Amador-Borrero B, et al. Clinical course of coronavirus disease 2019 (COVID-19) in a series of 17 patients with systemic lupus erythematosus under long-term treatment with hydroxychloroquine. Ann Rheum Dis. 2020;79:837-9.doi: 10.1136/annrheumdis-2020-217566

16. Gianfrancesco M, Hyrich KL, Al-Adely S, Carmona L, Danila MI, Gossec L, et al. Characteristics associated with hospitalisation for COVID-19 in people with rheumatic disease: data from the COVID-19 Global Rheumatology Alliance physician- reported registry. Ann Rheum Dis. 2020;79:859.doi: 10.1136/ annrheumdis-2020-217871

17. Gianfrancesco MA, Hyrich KL, Gossec L, Strangfeld A, Carmona L, Mateus EF, et al. Rheumatic disease and COVID-19: initial data from the COVID-19 global rheumatology alliance provider registries. The Lancet Rheumatology. 2020;2:e250-e3. doi: 10.1016/s2665-9913(20)30095-3

18. Monti S, Balduzzi S, Delvino P, Bellis E, Quadrelli VS, Montecucco C. Clinical course of COVID-19 in a series of patients with chronic arthritis treated with immunosuppressive targeted therapies. Ann Rheum Dis. 2020;79:667-8.doi: 10.1136/annrheumdis-2020-217424

19. Wu Z, McGoogan JM. Characteristics of and important lessons from the coronavirus disease 2019 (COVID-19) outbreak in China: summary of a report of 72314 cases from the Chinese Center for Disease Control and Prevention. JAMA. 2020;323:1239-42.doi: 10.1001/jama.2020.2648

20. Guan W-j, Ni Z-y, Hu Y, Liang W-h, Ou C-q, He J-x, et al. Clinical characteristics of coronavirus disease 2019 in China. $\mathrm{N}$ Engl J Med. 2020;382:1708-20.doi: 10.1056/NEJMoa2002032

21. Noreña I, Fernández-Ruiz M, Aguado JM. Viral infections in the biologic therapy era. Expert Rev Anti Infect Ther. 2018;16:78191. doi: 10.1080/14787210.2018.1521270

22. Shi $Y$, Wang $Y$, Shao $C$, Huang J, Gan J, Huang $X$, et al. COVID-19 infection: the perspectives on immune responses. Cell Death Differ. 2020;27:1451-4. doi: 10.1038/s41418-0200530-3. 